\title{
A neuroanatomical correlate of sensorimotor recovery in response to repeated vaginocervical stimulation in rats
}

\author{
Dina Conde ${ }^{1}$ and Barry R. Komisaruk ${ }^{2 *}$ \\ ' Department of Biology, Rutgers, The State University of New Jersey, Newark, NJ, USA \\ 2 Department of Psychology, Rutgers, The State University of New Jersey, Newark, NJ, USA
}

Edited by:

Charles Hubscher, University of

Louisville, USA

Reviewed by:

Noritaka Ichinohe, National Institute

of Neuroscience, National Center of

Neurology and Psychiatry, Japan

Lesley Marson, Urogenix Inc., USA

${ }^{*}$ Correspondence:

Barry R. Komisaruk, Department of

Psychology, Rutgers, The State

University of New Jersey, 101 Warren

Street, Newark, NJ 07102, USA.

e-mail: brk@psychology.rutgers.edu
Gentle probing against the cervix via the vagina (vaginocervical stimulation, VCS) increases tail flick latency (TFL) to radiant heat; greater force abolishes the tail flick response and other withdrawal responses. This effect occurs in spinal cord-transected rats and in intact rats. On the basis of our earlier finding that VCS releases vasoactive intestinal peptide (VIP) into the spinal cord, and others' reports of neurotrophic effects of VIP in vitro, we hypothesized that repeated VCS would stimulate sprouting and sensorimotor function of terminals of genital nerve primary afferents in the sacral spinal cord. To test this hypothesis, in the present study, we denervated the genital tract only unilaterally, which significantly reduced the TFL-elevating effect of VCS. Then we applied repeated daily VCS for 1 week and compared the subsequent effectiveness of acute VCS in elevating TFL. The rats that received the repeated daily VCS showed a significantly greater elevation in TFL in response to acute VCS than control rats that did not receive the repeated stimulation. Then, to test whether daily repeated VCS stimulates sprouting of genital primary afferents in such unilaterally genital tract-denervated rats, we transected the contralateral remaining intact pelvic nerve, applied horseradish peroxidase (HRP) to its proximal cut end for 1-2 $h$, and 2-3 days later counted HRP particles in its terminal zone (L6-S1) in the spinal cord. There were significantly more HRP particles in the rats that received the daily repeated VCS than in the control rats. In the context of these findings, we conclude that VCS in rats can produce a functional sensorimotor recovery via a neurotrophic effect on compromised primary afferents in the spinal cord.

\section{Keywords: recovery of function, genital stimulation, sprouting, primary afferents, HRP, analgesia}

\section{INTRODUCTION}

Vaginocervical stimulation (VCS) in rats acts at the spinal cord to block withdrawal reflexes to noxious stimulation. Hind leg and tail withdrawal reflexes, which persisted after surgical removal of a segment of the spinal cord at the mid-thoracic level, were still completely blocked by VCS (Komisaruk and Larsson, 1971). A possible mechanism for this effect is that foot-shock-induced release of substance $\mathrm{P}$ into spinal cord superfusate is inhibited by concurrent VCS (Steinman et al., 1994). The pelvic nerves, which provide sensory innervation of the vagina and cervix (Komisaruk et al., 1972; Peters et al., 1987; Berkley et al., 1990, 1993) are the main mediator of this VCS-induced reflex inhibition, for transection of the pelvic nerves almost completely abolishes the ability of VCS to block withdrawal reflexes (Cunningham et al., 1991). Vasoactive intestinal peptide (VIP) is a possible mediator of this effect of VCS, for pelvic nerve contains VIP (Basbaum and Glazer, 1983), VCS releases VIP into spinal cord superfusates (Komisaruk et al., 1989 ), and VIP and certain of its fragments administered directly to the spinal cord, intrathecally, mimic the analgesia-producing effects of VCS (Komisaruk and Jordan, 1995). And in a different context, VIP exerts neurotrophic effects (Brenneman and Eiden, 1986; Gozes et al., 1991; Gressens et al., 1993; Brenneman et al., 1999).

These findings combined led us to hypothesize that VCS could promote recovery of spinal cord-mediated behavioral function. To test this hypothesis, we first reduced the inhibitory effect of VCS on the tail flick latency (TFL) to radiant heat test by transecting the pelvic nerve unilaterally. In order to maximize the unilateral genital neurectomy effect, we also ipsilaterally transected the other genital sensory nerves, i.e., the pudendal and hypogastric (Komisaruk etal., 1972; Peters et al., 1987; Berkley et al., 1990, 1993). Then we applied repeated VCS three times daily for 1 week. We reasoned that the daily repeated VCS would stimulate sprouting of the remaining intact pelvic nerve terminals, and thereby increase the effectiveness of the VCS. Thus, in the present study, we ascertained whether: (a) unilateral genital neurectomy would reduce the magnitude of VCS-induced inhibition of the TFL, (b) daily repeated VCS would overcome this attenuation, and (c) the daily repeated VCS would induce sprouting of the remaining intact pelvic nerve terminals.

\section{MATERIALS AND METHODS EXPERIMENT 1. DOES DAILY REPEATED VCS IN GENITAL UNILATERAL-NEURECTOMIZED RATS INCREASE ITS INHIBITORY EFFECT ON TFL? Subjects}

Sprague Dawley female rats at least 90 days of age and weighing 300-400 g, were purchased from Charles River Breeding Laboratories. All animals were maintained on a reversed-light cycle 
(lights on 7:00 p.m. to 10:00 a.m.) with food and water ad libitum. Rats were housed two per cage in tubs with absorbent material flooring. All rats were ovariectomized at least 1 month prior to nerve transections to eliminate variability in response to VCS due to fluctuating levels of ovarian hormones (Crowley et al., 1976).

\section{Groups}

Animals were randomly assigned to the following groups: C: shamoperated controls, $n=10$; GenX: unilateral pelvic-hypogastricpudendal neurectomy, $n=11$; GenX + VCS: unilateral pelvichypogastric-pudendal neurectomy receiving three times daily VCS, $n=11$; C + VCS: intact controls receiving three times daily VCS, $n=12$.

\section{Surgery}

All experimental procedures used in the present study received approval from the Rutgers University Institutional Animal Care and Use Committee. Nerve transections: rats were anesthetized with a solution of $0.5 \mathrm{ml}$ Rompun/10 ml ketamine; dose: $0.07 \mathrm{ml} / 100 \mathrm{~g}$ body weight. A ventral abdominal incision, approximately $6 \mathrm{~cm}$ length, exposed the abdominal-pelvic region and facilitated the identification of the genitospinal nerves.

Unilateral hypogastric neurectomy. We identified the left ureter by its vigorous peristaltic activity, then visualized the left hypogastric nerve between the colon and the ureter as it courses parallel to the ureter, and removed a $4 \mathrm{~mm}$ segment of the nerve.

Unilateral pelvic neurectomy. The bifurcation of the vena cava into the common iliac veins was identified, the left common iliac vein was followed caudad, approximately $1 \mathrm{~cm}$, to the origin of the left internal iliac vein. Approximately $5 \mathrm{~mm}$ dorsal to the origin of the left internal iliac vein, perpendicular to its axis, we identified the left pelvic nerve as it courses rostrocaudad, lying across the vein, and removed a $4 \mathrm{~mm}$ segment.

Unilateral pudendal neurectomy. The left pudendal nerve was located dorsal and parallel to the left pelvic nerve as it, also, lies across the internal iliac vein. The pudendal nerve is wellmyelinated and glistens, relative to the pelvic nerve. A $4 \mathrm{~mm}$ segment was removed.

Sham neurectomy. The hypogastric, pelvic, and pudendal nerves were located unilaterally, and then each nerve was gently lifted with a microsurgical hook and returned to its original site.

\section{Behavioral testing}

Baseline TFL responses to VCS were obtained 1 week prior to neurectomy or control procedures. Each rat was placed in a hemi-cylindrical Plexiglas restrainer for approximately $5 \mathrm{~min}$ prior to testing. TFL responses before and during VCS (100 and $300 \mathrm{~g}$ force) were established prior to neurectomy or sham surgery.

TFL test. This measures the latency (in s) to flick the tail away from a focused regulated radiant heat source. The radiant heat source (IITC Inc., Model 33) was located $8 \mathrm{~cm}$ above and $4 \mathrm{~cm}$ proximal to the tip of the tail. The temperature of the heat lamp of the tail flick apparatus was adjusted to elicit a group mean approximate TFL of $3 \mathrm{~s}$. The TFL reading displayed automatically when the rat withdrew the tip of its tail away from the radiant heat source, breaking a photocell circuit. To prevent tissue damage, the heat source was stopped at $9 \mathrm{~s}$ if the rat did not withdraw its tail. Each test consisted of three trials $15 \mathrm{~s}$ apart. The score for each test was calculated as the mean latency of three successive trials.

Acute VCS. VCS (100 and $300 \mathrm{~g}$ force) was applied to the cervix using a calibrated "Force Dial Hand Held Dynamometer" (Model FD 500, Wagner Instruments, Greenwich, CT, USA). The tip of the metal plunger of the force-calibrated dynamometer was shaped with a dental sanding disk in order to match the tip of a $1 \mathrm{cc}$ plastic syringe plunger. To provide cushioning during VCS, we placed the rubber tip of a $1 \mathrm{ml}$ plastic syringe onto this modified tip.

Daily repeated VCS began $18 \mathrm{~h}$ after unilateral neurectomies and continued for 1 week. VCS (100 $g$ force, $10 \mathrm{~s}$ on, $10 \mathrm{~s}$ off $\times 5 \mathrm{~min}$ ) was applied three times daily, $2 \mathrm{~h}$ apart. The effect of acute VCS on TFL (100 and $300 \mathrm{~g}$ force) was ascertained 1 week after the daily VCS treatment ended, using a blind procedure.

\section{Statistical analysis}

The data are expressed as mean \pm SEM. Comparison among groups was made using repeated measures, Two-way analysis of variance (ANOVA), and subsequent Fisher's protected $t$-tests.

\section{EXPERIMENT 2. DOES DAILY REPEATED VCS INDUCE SPROUTING IN GENITAL PRIMARY AFFERENT NERVE TERMINALS? Subjects}

Eight Sprague Dawley female rats were purchased from Charles River Breeding Laboratories, at least 90 days of age and weighing 250-400 $\mathrm{g}$ at the time of surgery. They were ovariectomized at least 2 weeks before surgery and maintained on a reversed-light cycle (lights on 7:00 p.m. to 10:00 a.m.) with food and water ad libitum.

\section{Groups}

Animals were randomly assigned to the following groups: Control group: the genitospinal nerves (hypogastric, pelvic, and pudendal nerves) were transected unilaterally (right side) as described above, $n=5$; VCS-treated group: the genitospinal nerves were transected unilaterally (right side). Then, beginning $18 \mathrm{~h}$ after unilateral neurectomies VCS (100 $g$ force) was applied for $5 \mathrm{~min}$ (10 s on/10 s off), $2 \mathrm{~h}$ apart, three times a day for 1 week, $n=3$. Two weeks post-surgery, in both the control and VCS-treated groups, the remaining intact contralateral nerve (pelvic nerve) was transected and the proximal end was dipped into horseradish peroxidase (HRP) (see details of method below). Two to three days after the nerve dip, the animals were sacrificed and the density of HRP particles in the terminal field of the nerve in the spinal cord was determined.

\section{Pelvic nerve transection and pelvic nerve dip}

Fourteen days post-lesion, the rats were anesthetized as above and the left pelvic nerve was exposed at the pelvic ganglion level and transected with microscissors. Approximately $5 \mu \mathrm{l} \mathrm{HRP} \mathrm{dissolved}$ in saline $(50 \mathrm{U} / \mu \mathrm{l})$ was used to fill a small cup made by cutting PE-10 tubing to fit the nerve and closing one end with petroleum 
jelly. The cut end of the proximal pelvic nerve was placed into the cup and exposed to the tracer for $1-2 \mathrm{~h}$. At the end of this period, the cup was gently removed from the pelvic nerve end and the incision sutured. The animals were then allowed to survive for 2-3 days.

\section{Perfusion and tissue preparation}

Two to three days after the nerve dip, the animals were deeply anesthetized as described above, and perfused, via the ascending aorta, with physiological saline at room temperature $(50 \mathrm{ml} / \mathrm{rat})$, followed by a solution of $1 \%$ paraformaldehyde and $1.25 \%$ glutaraldehyde in $0.1 \mathrm{M}$ phosphate buffer at room temperature (500 $\mathrm{ml} / \mathrm{rat}$ ). After perfusion, the L6-S1 spinal cord segments were identified and removed, placed in a sucrose-buffer solution at $4^{\circ} \mathrm{C}$ for $12-24 \mathrm{~h}$ and cut into $30 \mu \mathrm{m}$ sections using a cryostat.

\section{Horseradish peroxidase histochemistry}

The mounted sections were processed for HRP histochemistry by using tetramethylbenzidine as the chromogen and stabilized with sodium nitroprusside according to the method of Mesulam and Brushart (1979). The sections were then air-dried overnight, cleared in hemoD, coverslipped with Permount mounting medium and examined under a Nikon light microscope using darkfield illumination. In all cases, the investigator making the histological observations was blind as to the treatments that corresponded to the slides. All sections L6-S1 were examined for the presence of HRP particles. HRP particles (reaction product granules) were identified and counted manually using the software program, "Neurolucida," under darkfield illumination. Spinal cord regions were identified and delineated according to Rexed's laminae, and the area of each region determined with the software program, "Neuroexplora." The selection of these sections was based on the presence of maximal HRP label intensity at the sacral parasympathetic nucleus (SPN) as observed under darkfield microscopy. The average of three sections (L6-S1) per animal was used to measure the density of HRP particles for each spinal cord region. HRP particle density was calculated by counting the total number of HRP reaction product granules and then dividing this value by the area of the corresponding spinal cord region. The images of the selected sections were analyzed using a Kodalith threshold that was adjusted for each image after manually identifying HRP particles and excluding background levels and artifacts. The average particle size was set at $\left(0.01-5 \mu \mathrm{m}^{2}\right)$ and the average size of $3 \mu \mathrm{m}$ was used to separate and thereby identify individual particles.

\section{Statistical analysis}

The data are expressed as the medians and interquartile ranges. The groups were compared using Mann-Whitney $U$-tests.

\section{RESULTS}

EXPERIMENT 1. EVIDENCE THAT DAILY REPEATED VCS INCREASES THE INHIBITORY EFFECT OF VCS ON TAIL FLICK

As summarized in Figure 1, all groups showed equivalent baseline (pre-VCS; "0 $g$ force") TFLs. As anticipated, the TFL in response to acute VCS (100 or $300 \mathrm{~g}$ force) was significantly lower in the unilateral genital neurectomy (GenX) group than in the intact

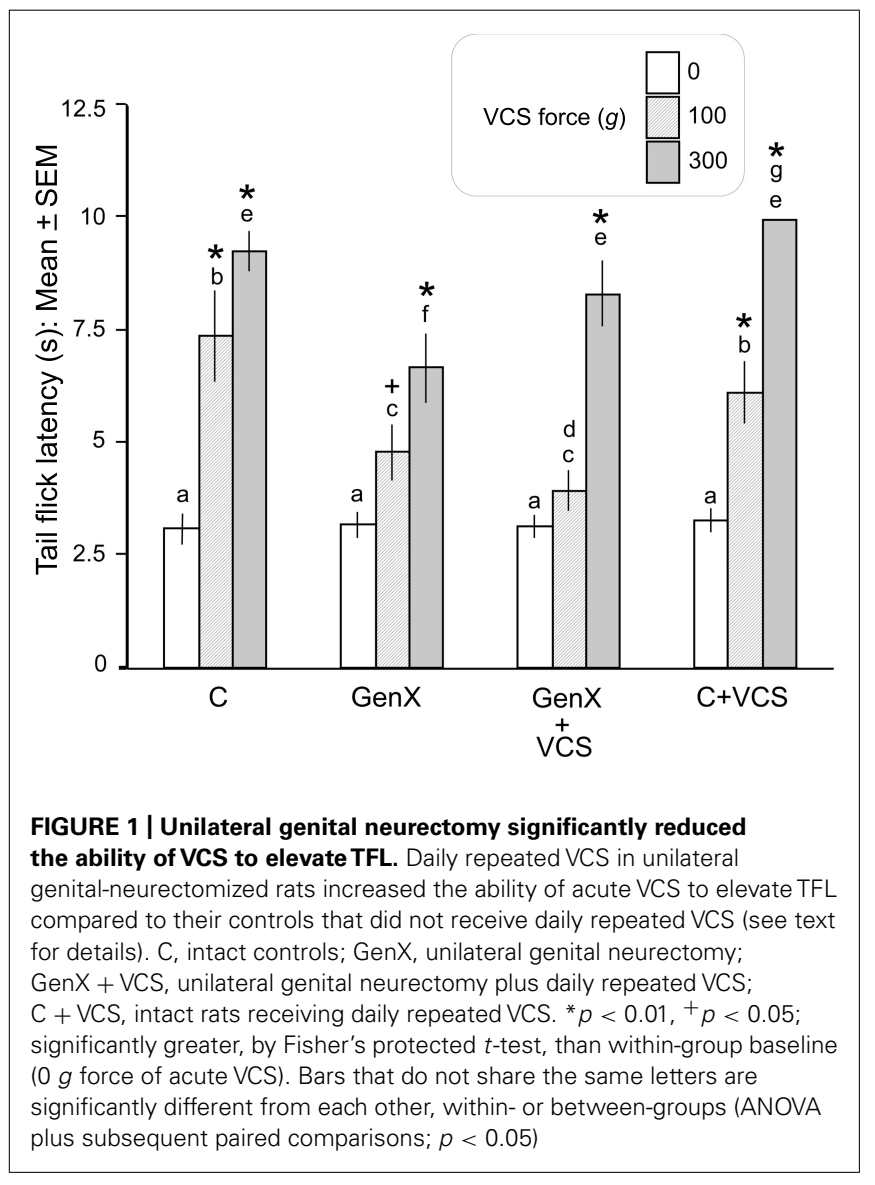

control (C) group ( $p<0.01$, Fisher's protected $t$-tests). Thus, the unilateral neurectomy significantly reduced the ability of VCS to inhibit the tail flick response. The TFL elevation to $300 \mathrm{~g}$ (although not $100 \mathrm{~g}$ ) VCS was significantly higher in the unilateral neurectomy + daily VCS group (GenX + VCS) than in the unilateral neurectomy group that did not receive daily VCS (GenX). However, in the intact group, daily repeated VCS $(\mathrm{C}+\mathrm{VCS})$ did not increase the ability of acute VCS to increase TFL any more than in the intact group that did not receive daily repeated VCS (C). Thus, the higher VCS force revealed the effect of daily repeated VCS to increase the ability of VCS to inhibit the tail flick response in the genital-neurectomized rats.

\section{EXPERIMENT 2. EFFECT OF UNILATERAL NERVE TRANSECTION AND DAILY REPEATED VCS ON HRP LABELING OF PELVIC NERVE TERMINALS}

HRP reaction product was located in the L6-S1 segments of the spinal cord. The primary afferent fibers enter this region through Lissauer's tract, diverging into a medial and a lateral band surrounding the superficial border of the dorsal horn. The collaterals of some primary afferents that course along the lateral band approach, and form connections with, SPNs and dendrites. Other collaterals course among SPNs and dendrites to form a terminal field in the upper dorsal gray commissure with fewer fibers crossing to the contralateral side of the spinal cord. The primary afferent collaterals emerging from the medial band form 


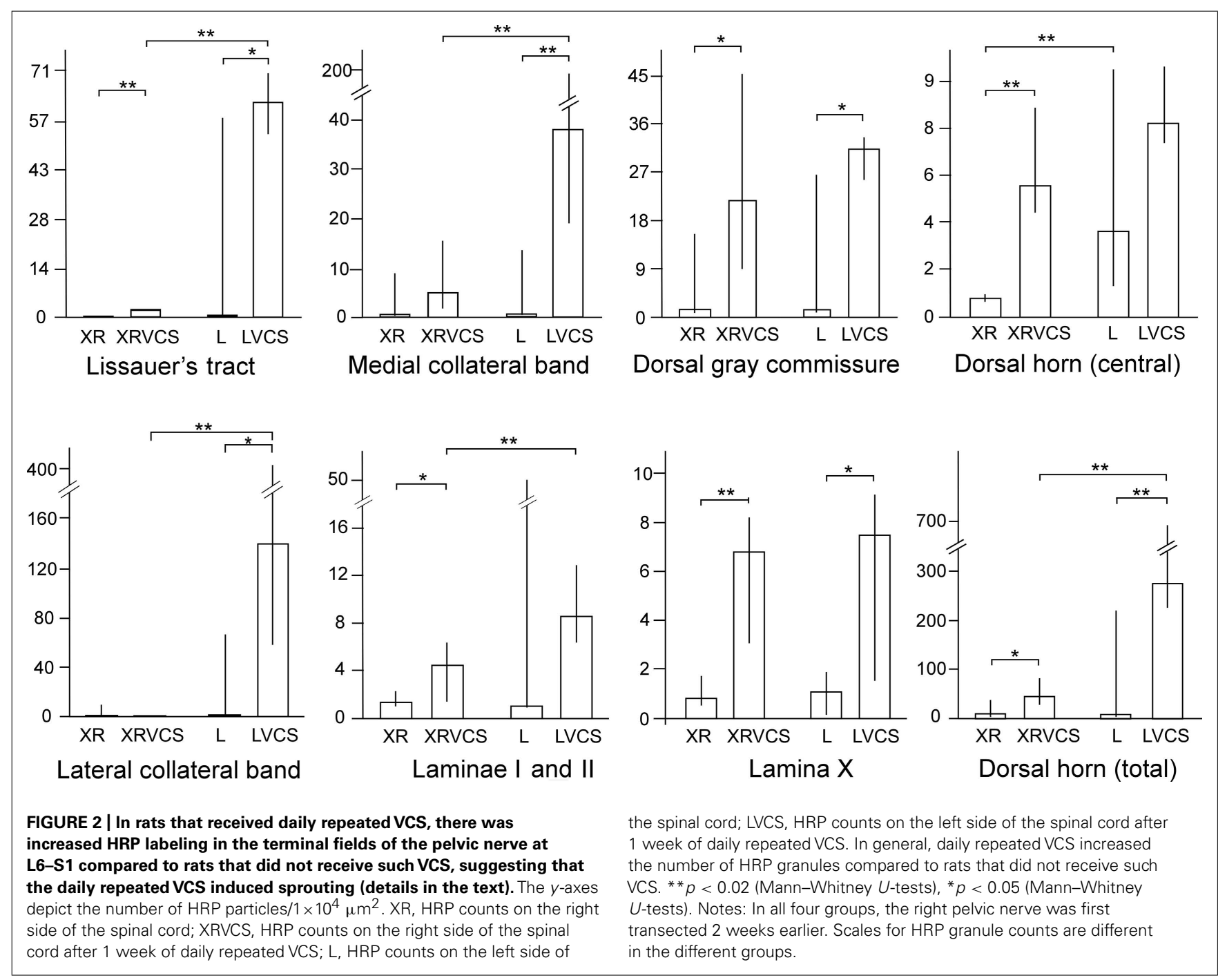

a terminal field in the upper dorsal gray commissure. Primary afferent collaterals/terminals are also located in Laminae I, II, and $\mathrm{X}$ (Figure 3).

\section{EVIDENCE THAT DAILY REPEATED VCS INCREASES HRP LABELING}

The purpose of transecting the genitospinal nerves unilaterally (on the right side) was to clear a field for the ipsilateral and contralateral terminals of the remaining contralateral pelvic nerve to potentially proliferate. As can be seen in Figure 2, in the nonVCS group (XR and L), there was minimal HRP labeling in either the ipsilateral or the contralateral side of the spinal cord in the regions specified. By contrast, in the VCS group (XRVCS and LVCS), there was significantly more labeling than in the non-VCS group in six of the eight ipsilateral (i.e., left side) intact regions. And in six of the eight neurectomized (i.e., right side) regions, there was significantly more labeling in the XRVCS group than in the non-VCS (XR) group. Thus, whereas relatively minimal HRP labeling occurred in the terminals in the absence of VCS, there was a marked increase in HRP labeling on both the ipsilateral and the contralateral sides in the group that received daily repeated
VCS. Table 1 summarizes the median number of HRP particles observed in each region of the spinal cord.

\section{DISCUSSION}

In the present study, unilateral denervation of the reproductive tract significantly reduced the magnitude of VCS-induced inhibition of the tail flick response to radiant heat. Daily repeated VCS for 1 week then significantly increased (restored) its analgesic effectiveness in these unilateral genital-denervated rats, whereas in the intact control rats, the same type of VCS did not increase further the analgesia magnitude. We tested a possible morphological basis for this effect by first transecting the pelvic nerve on the right side in order to vacate its terminal projection field. Subsequently, we applied HRP to the acutely cut proximal end of the remaining intact pelvic nerve on the left side, and then counted HRP reaction product in the pelvic nerve terminal projection zone at spinal cord level L6-S1 (Nadelhaft and Booth, 1984). The present findings provide support for the interpretation that daily repeated VCS increased the terminal proliferation, on the basis that the daily repeated VCS significantly and markedly increased the counts of 


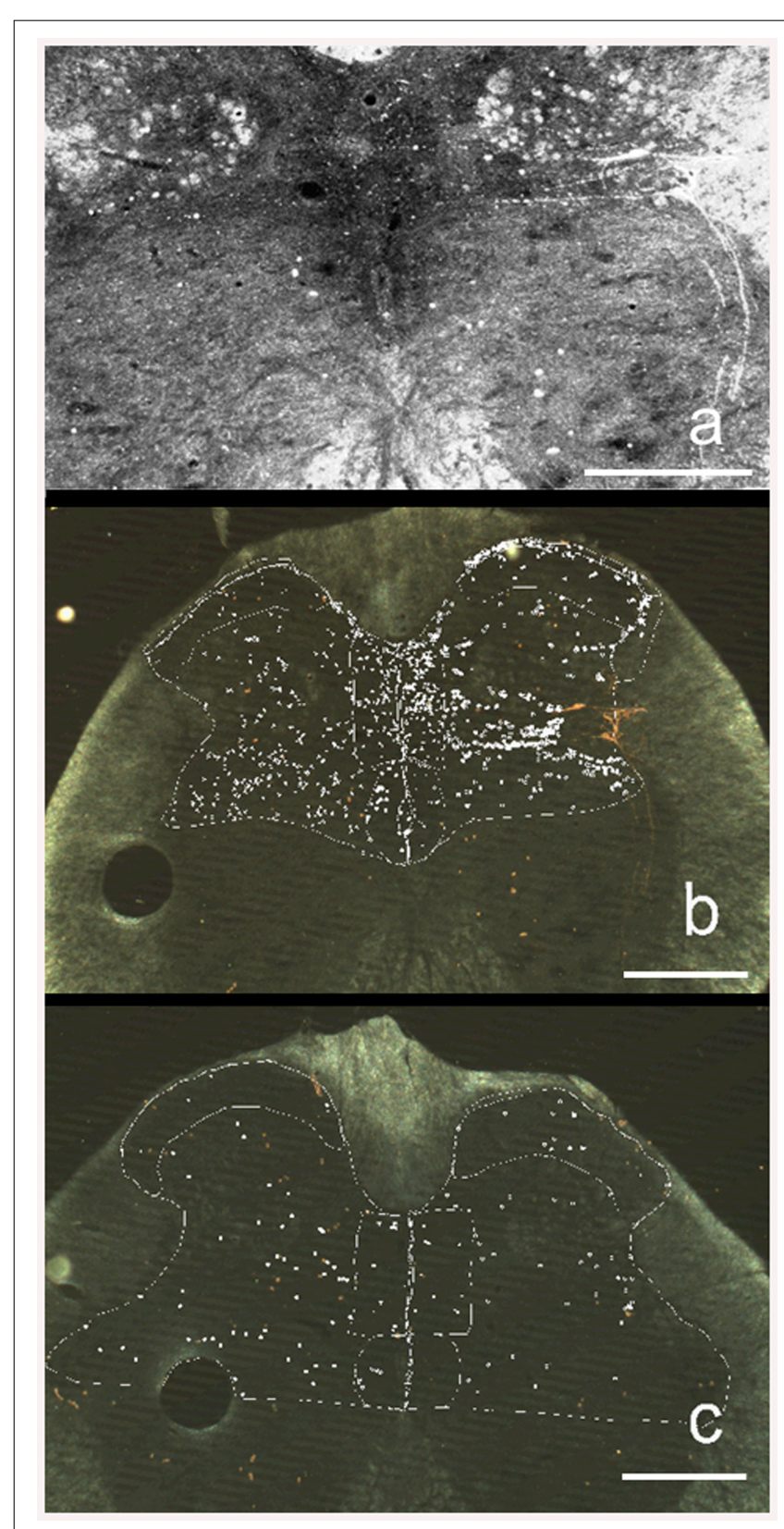

FIGURE 3 | Darkfield illumination photomicrograph (Neurolucida) of transverse $30-\mu \mathrm{m}$ sections of the sacral 1 (S1) segment of the spinal cord. The right side of the figures is the left side of the spinal cord. (a) HRP labeling in a rat that received daily repeated VCS. Brightened image to emphasize appearance and location of HRP particles (white dots). (b) Same section as above showing how HRP counting was made. The white marker dots indicate the locations of HRP particles. Different types of marker dots were used to differentiate the selected regions of the spinal cord. (c) HRP labeling in a control rat that did not receive daily repeated VCS. Calibration bar $=200 \mu$

HRP reaction product in the pelvic nerve terminal field in six of the eight ipsilateral terminal fields measured, as well as in six of the eight contralateral terminal fields measured (The pelvic nerve distributes its terminals ipsi- and contra-laterally; Basbaum and Glazer, 1983; Chung et al., 1993).
Table 1 | Regional distribution of median number of HRP particles $/ 1 \times 10^{4} \mu \mathrm{m}^{2}$.

\begin{tabular}{lllll}
\hline Spinal cord region & XR & XRVCS & L & LVCS \\
\hline Lissauer's tract & 0 & 1.88 & 0 & 61.33 \\
Medical collateral band & 0 & 4.47 & 0 & 37.76 \\
Dorsal gray commissure & 1.15 & 21.38 & 0.90 & 30.88 \\
Dorsal horn (central) & 0.70 & 5.19 & 3.39 & 7.73 \\
Lateral collateral band & 0 & 0 & 0 & 138.09 \\
Laminae I and II & 1.20 & 4.33 & 0.90 & 8.55 \\
Lamina X & 0.86 & 6.64 & 1.04 & 7.43 \\
Dorsal horn (total) & 4.04 & 44.01 & 5.12 & 273.47 \\
\hline
\end{tabular}

This is a numerical summary of the data shown in Figure 2. In the control group $(n=5)$, the right genitospinal (hypogastric, pelvic, pudendal) nerves were transected (XR) 2 weeks before HRP administration to the intact contralateral pelvic nerve [left side of the spinal cord $(L)]$. In the experimental group $(n=3)$ rats were treated with a daily regimen of VCS following unilateral right side genitospinal nerve transection (XRVCS). Following daily VCS treatment HRP was administered to the intact contralateral pelvic nerve (LVCS). HRP counts were done in both sides of the spinal cord (see text for details).

The validity of the quantitative HRP method used in the present study is supported by our finding of a distribution of primary afferent fiber terminals consistent with the distribution described by others (Nadelhaft and Booth, 1984). According to those authors, pelvic nerve primary afferent fibers enter Lissauer's Tract in the spinal cord in the same segments (L6-S2) as those in which the preganglionic neurons are located that enter the pelvic nerve efferents. This supports our criterion for selecting, for quantitative HRP analysis, sections with SPN neurons showing maximal HRP label, since these sections also contain primary afferent fibers and terminals of the pelvic nerve.

We speculate that the marked quantitative increase in HRP reaction product in the pelvic nerve terminal fields in the daily repeated VCS group represents VCS-induced proliferation, i.e., sprouting, of pelvic nerve primary afferent terminals in the spinal cord, thereby mediating the ability of repeated daily VCS to increase its tail flick response-inhibiting potency.

A mechanism for this effect may be the release of neurotrophic substances into the dorsal horn of the spinal cord, inducing sprouting of intact pelvic nerve fibers into the denervated region. Sprouting occurs spontaneously within 2 weeks of trauma (Woolf et al., 1995); the present findings suggest that daily repeated VCS can markedly intensify this process. Several other mechanisms could play a role in functional recovery of the CNS in response to injury, e.g., a rearrangement of spared neuronal circuits, an upregulation of neuropeptides with neurotrophic abilities (Muir and Steeves, 1997), and/or an acceleration of transport of neurotrophic substances to the terminal fields, perhaps represented by HRP accelerated transport of HRP particles. VCS, which activates the pelvic nerve, releases VIP into the spinal cord (Komisaruk et al., 1989). Evidence that VIP is a neurotrophic factor is that it induces neurite growth in vitro and protects neurons from cell death (Brenneman and Eiden, 1986; Pincus et al., 1990; White and Mansfield, 1996). VIP increases neuronal survival indirectly, via the release of several neurotrophic substances from glial cells 
(Brenneman et al., 1987, 1990). VIP exerts neurotrophic effects on developing neurons in the CNS (Brenneman and Eiden, 1986); dissociated cell cultures and whole embryo cultures showed a marked increase in growth markers in response to VIP treatment (Gressens et al., 1993); and VIP increases the survival of electrically blocked spinal neurons (Brenneman and Eiden, 1986; Brenneman etal., 1999). Also, blocking the action of VIP with neutralizing VIP antisera or with VIP receptor antagonists resulted in neuronal cell death for a subpopulation of CNS neurons in spinal cord, hippocampus, and cerebral cortex (Brenneman and Eiden, 1986; Gozes et al., 1991).

These findings led us to speculate that VCS, by releasing VIP into the spinal cord, could promote the recovery of spinal cordmediated behavioral function for which evidence is presented in the present study.

Because the TFL test measures a motor response, from this test one cannot distinguish whether the inhibitory effect of VCS on the tail flick response is on the motor response only, nociception only, or both. However, there is substantial evidence that VCS does at least produce analgesia. That is, in rats VCS inhibits nociceptive responses in thalamic sensory neurons (Komisaruk and Wallman, 1977), and in women vaginal/cervical self-stimulation produces a marked elevation in pain thresholds (Komisaruk and Whipple, 1984; Whipple and Komisaruk, 1985). Those effects are analgesic, rather than anesthetic, because in both cases, responses to innocuous tactile stimulation were not affected by the VCS.

The present study focuses on the proliferation of primary afferent pelvic nerve terminals in the spinal cord in response to daily repeated VCS and the resulting increase in the analgesic effect of VCS. While this process involves an active inhibitory process within the spinal cord, there is evidence that a parallel supraspinal process is also activated by VCS. That is, in women with complete

\section{REFERENCES}

Basbaum, A. I., and Glazer, E. J. (1983). Immunoreactive vasoactive intestinal polypeptide is concentrated in the sacral spinal cord: a possible marker for pelvic visceral afferent fibers. Somatosens. Res. 1, 69-82.

Berkley, K. J., Hotta, H., Robbins, A., and Sato, Y. (1990). Functional properties of afferent fibers supplying reproductive and other pelvic organs in pelvic nerve of female rats. $J$. Neurophysiol. 63, 256-272.

Berkley, K. J., Robbins, A., and Sato, Y. (1993). Functional differences between afferent fibers in the hypogastric and pelvic nerves innervating female reproductive organs in the rat. J. Neurophysiol. 69, 533-544.

Brenneman, D. E., and Eiden, L. E. (1986). Vasoactive intestinal peptide and electrical activity influence neuronal survival. Proc. Nat. Acad. Sci. U.S.A. 83, 1159-1162.

Brenneman, D. E., Hauser, J., Phillips, T. M., Davidson, A., Bassan, M., and Gozes, I. (1999). Vasoactive intestinal peptide. Link between electrical activity and glia-mediated neurotrophism. Ann. N. Y. Acad. Sci. 897, 17-26.

Brenneman, D. E., Neale, E. A., Foster, G. A., d'Autremont, S. W., and Westbrook, G. L. (1987). Nonneuronal cells mediate neurotrophic action of vasoactive intestinal peptide. J. Cell Biol. 104, 1603-1610.

Brenneman, D. E., Nicol, T., D., Warren, D., and Bowers, L. M. (1990). Vasoactive intestinal peptide: a neurotrophic releasing agent and an astroglial mitogen. J. Neurosci. Res. 25, 386-394.

Chung, K., Lee, W. T., and Park, M. J. (1993). Spinal projections of pelvic visceral afferents of the rat: a calcitonin gene-related peptide (CGRP) immunohistochemical study. J. Comp. Neurol. 337, 63-69.

Crowley, W. R., Jacobs, R., Volpe, J., Rodriguez-Sierra, J. F., and Komisaruk, B. R. (1976). Analgesic effect

spinal cord injury at T10 or above, we previously showed that vaginal or cervical self-stimulation produces analgesia, evidenced by significant increases in pain thresholds to calibrated compressive force applied to the fingers (Komisaruk et al., 1997). Evidence that this analgesia is mediated by the vagus nerves is that by using functional MRI in these women, we found that the vaginal or cervical self-stimulation activated the terminal projection zone of the vagus nerves in the medulla oblongata, i.e., the nucleus of the solitary tract (Komisaruk et al., 2004). Thus, the vagus nerves (cranial nerve 10) provide a previously unrecognized genital sensory pathway directly to the brain, bypassing the spinal cord. And stimulation of this nerve via the vagina or cervix can produce analgesia.

On the basis of the present findings that daily repeated VCS can increase the analgesic action of VCS by proliferating pelvic nerve terminals in the spinal cord, it is tempting to speculate that the same stimulus could stimulate the proliferation of vagus nerve terminals above complete spinal cord injury at any level, for the terminal fields of genital afferents ascending to the brain via the spinal cord would also be vacated by the de-afferentation. An intriguing possibility is whether, via the vagus nerve afferents, in women with "complete" spinal cord injury at or above T10, vaginal or cervical self-stimulation could attenuate pain that is perceived above - and, via the vagus nerve pathway even below - the level of complete spinal cord injury, and whether repeated VCS in women could augment the attenuation of such pain.

\section{ACKNOWLEDGMENTS}

We gratefully acknowledge Professor Irving Nadelhaft for his instruction and help in utilizing the HRP methodology, and Professor Laszlo Zaborszky for his gracious guidance and providing the use of his histological facility for computerized quantitative analysis of the HRP data.

of vaginal stimulation in rats: modulation by graded stimulus intensity and hormones. Physiol. Behav. 16, 483-488.

Cunningham, S. T., Steinman, J. L., Whipple, B., Mayer, A. D., and Komisaruk, B. R. (1991). Differential roles of hypogastric and pelvic nerves in the analgesic and motoric effects of vaginocervical stimulation in rats. Brain Res. 559, 337-343.

Gozes, I., McCune, S. K., Jacobson, L., Warren, D., Moody, T. W., Fridkin, M., and Brenneman, D. E. (1991). An Antagonist to vasoactive intestinal peptide affects cellular functions in the central nervous system. $J$. Pharmacol. Exp. Ther. 257, 959-966.

Gressens, P., Hill, J. M., Gozes, I., Fridkin, M., and Brenneman, D. E. (1993). Growth factor function of vasoactive intestinal intestinal peptide in whole cultured mouse embryos. Nature 362, 155-158.

Komisaruk, B. R., Adler, N. T., and Hutchison, J. (1972). Genital sensory field: enlargement by estrogen treatment in female rats. Science 178, 1295-1298.

Komisaruk, B. R., Gerdes, C. A., and Whipple, B. (1997). "Complete" spinal cord injury does not block perceptual responses to genital selfstimulation in women. Arch. Neurol. 54, 1513-1520.

Komisaruk, B. R., Gintzler, A. R., Banas, C., and Blank, M. S. (1989). Vaginocervical stimulation releases vasoactive intestinal peptidelike immunoreactivity (VIP) into spinal cord superfusates in rats. Soc. Neurosci. Abstr. 15, 216.

Komisaruk, B. R., and Jordan, F. (1995). Process for inducing analgesia, peptides and therapeutic compositions. Patent \# 5,426,099. U.S. Patent Office.

Komisaruk, B. R., and Larsson, K. (1971). Suppression of a spinal and a cranial nerve reflex by vaginal or rectal probing in rats. Brain Res. 35 , 231-235.

Komisaruk, B. R., and Wallman, J. (1977). Antinociceptive effects of 
vaginal stimulation in rats: neurophysiological and behavioral studies. Brain Res. 137, 85-107.

Komisaruk, B. R., and Whipple, B. (1984). Evidence that vaginal selfstimulation in women suppresses experimentally-induced finger pain. Soc. Neurosci. Abstr. 10, 675

Komisaruk, B. R., Whipple, B., Crawford, A., Grimes, S., Liu, W.-C., Kalnin, A., and Mosier, K. (2004). Brain activation during vaginocervical self-stimulation and orgasm in women with complete spinal cord injury: fMRI evidence of mediation by the vagus nerves. Brain Res. 1024, 77-88.

Mesulam, M. M., and Brushart, T. M. (1979). Transganglionic and anterograde transport of horseradish peroxidase across dorsal root ganglia: a tetramethylbenzidine method for tracing central sensory connections of muscles and peripheral nerves. Neuroscience 4, 1107-1117.
Muir, G. D., and Steeves, J. D. (1997). Sensorimotor stimulation to improve locomotor recovery after spinal cord injury. Trends Neurosci. 20, 72-77.

Nadelhaft, I., and Booth, A. M. (1984). The location and morphology of preganglionic neurons and the distribution of visceral afferents from the rat pelvic nerve: a horseradish peroxidase study. J. Comp. Neurol. 226, 238-245.

Peters, L. C., Kristal, M. B., and Komisaruk, B. R. (1987). Sensory innervation of the external and internal genitalia of the female rat. Brain Res. 408, 199-204.

Pincus, D. W., DiCicco-Bloom, E. M., and Black, I. B. (1990). Vasoactive intestinal peptide regulates mitosis, differentiation and survival of cultured sympathetic neuroblasts. Nature 343, 564-567.

Steinman, J. L., Hoffman, S. W., Banas, C., and Komisaruk, B. R. (1994). Vaginocervical stimulation attenuates hindpaw shock-induced substance $\mathrm{P}$ release into spinal cord superfusates in rat. Brain Res. 647, 204-208.

Whipple, B., and Komisaruk, B. R. (1985). Elevation of pain threshold by vaginal stimulation in women. Pain 21, 357-367.

White, D. M., and Mansfield, K. (1996). Vasoactive intestinal peptide and neuropeptide $\mathrm{Y}$ act indirectly to increase neurite outgrowth of dissociated dorsal root ganglion cells. Neuroscience 73, 881-887.

Woolf, C. J., Shortland, P., Reynolds, M., Ridings, J., and Doubell, T. (1995). Reorganization of central terminals of myelinated primary afferents in the rat dorsal horn following peripheral axotomy. J. Comp. Neurol. 360, 121-134.

Conflict of Interest Statement: The authors declare that the research was conducted in the absence of any commercial or financial relationships that could be construed as a potential conflict of interest.

Received: 07 January 2012; paper pending published: 30 January; accepted: 30 March 2012; published online: 19 April 2012.

Citation: Conde D and Komisaruk BR (2012) A neuroanatomical correlate of sensorimotor recovery in response to repeated vaginocervical stimulation in rats. Front. Physio. 3:100. doi: 10.3389/ fphys.2012.00100

This article was submitted to Frontiers in Integrative Physiology, a specialty of Frontiers in Physiology.

Copyright () 2012 Conde and Komisaruk. This is an open-access article distributed under the terms of the Creative Commons Attribution Non Commercial License, which permits non-commercial use, distribution, and reproduction in other forums, provided the original authors and source are credited. 